九州大学学術情報リポジトリ

Kyushu University Institutional Repository

\title{
Suppression of the quantum-mechanical collapse by repulsive interactions in a quantum gas
}

Sakaguchi, Hidetsugu

Department of Applied Science for Electronics and Materials, Interdisciplinary Graduate School of Engineering Sciences, Kyushu University

Malomed, Boris A.

Department of Physical Electronics, School of Electrical Engineering, Faculty of Engineering, Tel Aviv University

http://hdl. handle. net/2324/25539

出版情報: Physical Review A : Atomic, Molecular, and Optical Physics. 83 (1), pp.013607(1)013607(7), 2011-01-14. American Physical Society

バージョン:

権利関係: (C) 2011 American Physical Society 


\title{
Suppression of the quantum-mechanical collapse by repulsive interactions in a quantum gas
}

\author{
Hidetsugu Sakaguchi ${ }^{1}$ and Boris A. Malomed ${ }^{2}$ \\ ${ }^{1}$ Department of Applied Science for Electronics and Materials, Interdisciplinary Graduate School of Engineering Sciences, \\ Kyushu University, Kasuga, Fukuoka 816-8580, Japan \\ ${ }^{2}$ Department of Physical Electronics, School of Electrical Engineering, Faculty of Engineering, Tel Aviv University, Tel Aviv 69978, Israel
}

(Received 15 March 2010; revised manuscript received 26 October 2010; published 14 January 2011)

\begin{abstract}
The quantum-mechanical collapse (alias fall onto the center of particles attracted by potential $-r^{-2}$ ) is a well-known issue in quantum theory. It is closely related to the quantum anomaly, i.e., breaking of the scaling invariance of the respective Hamiltonian by quantization. We demonstrate that the mean-field repulsive nonlinearity prevents the collapse and thus puts forward a solution to the quantum-anomaly problem that differs from that previously developed in the framework of the linear quantum-field theory. This solution may be realized in the 3D or 2D gas of dipolar bosons attracted by a central charge and in the 2D gas of magnetic dipoles attracted by a current filament. In the 3D setting, the dipole-dipole interactions are also taken into regard, in the mean-field approximation, resulting in a redefinition of the scattering length which accounts for the contact repulsion between the bosons. In lieu of the collapse, the cubic nonlinearity creates a 3D ground state (GS), which does not exist in the respective linear Schrödinger equation. The addition of the harmonic trap gives rise to a tristability, in the case when the Schrödinger equation still does not lead to the collapse. In the 2D setting, the cubic nonlinearity is not strong enough to prevent the collapse; however, the quintic term does it, creating the GS, as well as its counterparts carrying the angular momentum (vorticity). Counterintuitively, such self-trapped 2D modes exist even in the case of a weakly repulsive potential $r^{-2}$. The $2 \mathrm{D}$ vortical modes avoid the phase singularity at the pivot $(r=0)$ by having the amplitude diverging at $r \rightarrow 0$ instead of the usual situation with the amplitude of the vortical mode vanishing at $r \rightarrow 0$ (the norm of the mode converges despite of the singularity of the amplitude at $r \rightarrow 0$ ). In the presence of the harmonic trap, the 2D quintic model with a weakly repulsive central potential $r^{-2}$ gives rise to three confined modes, the middle one being unstable, spontaneously developing into a breather. In both the 3D and 2D cases, the GS wave functions are found in a numerical form and in the form of an analytical approximation, which is asymptotically exact in the limit of the large norm.
\end{abstract}

DOI: 10.1103/PhysRevA.83.013607

PACS number(s): 03.75.Kk, 03.75.Lm, 05.45.Yv

\section{INTRODUCTION}

It is well known that attractive potential $U(r)=$ $-\left(U_{0} / 2\right) r^{-2}$ plays a critical role in quantum mechanics. Indeed, while the corresponding Hamiltonian, taken in the scaled form, $\hat{H}=-(1 / 2) \nabla^{2}+U(r)$, obeys the scaling invariance,

$$
\hat{H}(\alpha r)=H(r) / \alpha^{2},
$$

the quantization breaks the invariance, which is known as the quantum anomaly, alias "dimensional transmutation" [1]. A manifestation of the anomaly is that, at $U_{0}>\left(U_{0}\right)_{\mathrm{cr}}^{(3 \mathrm{D})} \equiv$ $1 / 4$, the corresponding 3D (three-dimensional) Schrödinger equation, $i \psi_{t}=\hat{H} \psi$, does not produce the ground state (GS); instead, it gives rise to the quantum collapse, also known as fall onto the center [2]. In the 2D space, the critical value of the potential's strength is exactly zero, $\left(U_{0}\right)_{\mathrm{cr}}^{(2 \mathrm{D})}=0$, and in the $1 \mathrm{D}$ setting the same potential gives rise to a still stronger superselection effect, effectively splitting the 1D space into two disjoint subspaces, $x \gtrless 0$ [3].

A solution to the quantum-anomaly problem in the $3 \mathrm{D}$ case was proposed outside the realm of quantum mechanics, in terms of a linear quantum-field-theory description, which introduces a renormalization and imposes a GS with an arbitrary spatial scale [1]. The purpose of the present work is to demonstrate that a different solution to this problem can be obtained within the framework of the mean-field approach, taking into account the effective repulsive nonlinearity induced by collisions of particles trapped in the potential under the consideration. In the $3 \mathrm{D}$ setting, the usual cubic self-repulsive term will be sufficient for this, while in the $2 \mathrm{D}$ geometry a stronger quintic term is necessary. As a result, we will find the GS with a scale uniquely defined by the physical parameters. It will be demonstrated that the same nonlinearity is helpful too below the critical point, where it makes the GS normalizable [at $U_{0}<\left(U_{0}\right)_{\mathrm{cr}}^{(3 \mathrm{D}, 2 \mathrm{D})}$, the linear Schrödinger equation gives rise to GS wave functions with the divergent norm, see below].

The linear Schrödinger equation with the critical potential is

$$
i \psi_{t}=-\frac{1}{2}\left(\nabla^{2}+U_{0} r^{-2}-\Omega^{2} r^{2}\right) \psi,
$$

where the external trapping potential, $\left(\Omega^{2} / 2\right) r^{2}$, is included, too [4]. A physical realization of Eq. (2) in the 3D space is provided by molecules with a permanent electric dipole moment, $d$, interacting with charge $Q$ placed at the origin, which creates electric field $\mathbf{E}=Q \mathbf{r} / r^{3}$. As demonstrated in a recent experiment, the charged particle (ion) immersed into an ultracold gas may be kept at the central position by means of the laser-trapping technique [5]. Assuming that the orientation of the dipole is locked to the local field, i.e., $\mathbf{d}=\operatorname{sgn}(Q) d(\mathbf{r} / r)$, the respective interaction potential is $U(r)=-\mathbf{d} \cdot \mathbf{E}$, which corresponds to Eq. (2) with

$$
U_{0}=2|Q| d .
$$

This realization is relevant to the capture of electrons by dipolar molecules, which was studied in detail experimentally [6,7], and to Bose-Einstein condensates (BECs) formed by dipolar molecules, such as Li-Cs [8] or K-Rb [9]. We stress 
that we consider the gas of permanent dipoles, while, in the case of polarizable molecules, with $\mathbf{d}=\varepsilon \mathbf{E}$, where $\varepsilon$ is the polarizability, the attractive potential is different, $U=$ $-\varepsilon Q^{2} r^{-4}$.

If the gas of ultracold dipolar molecules is trapped in a pancake-shaped configuration sustained by an appropriate external potential [10], inserting the central electric charge provides for the realization of the $2 \mathrm{D}$ version of Eq. (2). An additional realization of the $2 \mathrm{D}$ setting is offered by a gas of atoms (chromium [11,12]) or molecules (such as ${ }^{87} \mathrm{Rb}_{2}$ [13]) carrying magnetic moments [14], the attractive potential being induced by the magnetic field of a transverse current filament or electron beam, with the orientation of the dipoles locked to the local magnetic field. Actually, the orientations of the dipoles may form the configurations obeying Eq. (2) in the dipolar BEC produced by means of the all-optical trapping [12], which does not freeze the dipoles into the confining magnetic field.

In the mean-field approximation, the contact repulsive interaction in the bosonic gas is represented by the cubic term [15]. With the addition of this term, the linear Schrödinger equation (2) is replaced by the Gross-Pitaevskii equation (GPE),

$$
i \psi_{t}=-(1 / 2)\left(\nabla^{2}+U_{0} r^{-2}-\Omega^{2} r^{2}\right) \psi+|\psi|^{2} \psi .
$$

The relation between the scaled variables and constants, in terms of which Eq. (4) is written, and their counterparts defined in the usual physical units [15] is

$$
\begin{gathered}
\mathbf{r}=\frac{\mathbf{r}_{\mathrm{ph}}}{r_{0}}, \quad t=\frac{\hbar}{m r_{0}^{2}} t_{\mathrm{ph}}, \quad \psi=2 \sqrt{\pi a_{s}} r_{0} \psi_{\mathrm{ph}}, \\
U_{0}=\frac{m}{\hbar^{2}}\left(U_{0}\right)_{\mathrm{ph}}, \quad \Omega=\frac{m r_{0}^{2}}{\hbar} \Omega_{\mathrm{ph}},
\end{gathered}
$$

where $m$ and $a_{s}$ are the bosonic mass and $s$-scattering length, which accounts for the repulsive interactions, and $r_{0}$ is an arbitrary spatial scale. Accordingly, the total number of bosons in the gas is given by

$$
N_{\mathrm{ph}}=\int\left|\psi_{\mathrm{ph}}\left(\mathbf{r}_{\mathrm{ph}}\right)\right|^{2} d \mathbf{r}_{\mathrm{ph}} \equiv \frac{r_{0} N}{4 \pi a_{s}},
$$

where $N=\int|\psi(\mathbf{r})|^{2} d \mathbf{r}$ is the norm of the scaled wave function. Note that, according to Eqs. (3) and (5), the abovementioned critical value of $U_{0}=1 / 4$ corresponds to a very small dipole moment, $d \sim 10^{-5}$ Debye, if $Q$ is taken as the elementary charge, and the mass of the particle is $\sim 10$ proton masses. Therefore, the case of $U_{0}>1 / 4$ is relevant one in particular physical contexts.

In the form of Eq. (4), the GPE neglects the dipoledipole interactions between the particles. However, these can be readily taken into account, using the same mean-field approximation which is used to derive the GPE. Indeed, the local density of the dipole moment in the gas (i.e., the polarization of the medium) is $\mathbf{P}=\mathbf{d}|\psi(\mathbf{r})|^{2}$, hence the additional electric field generated by the polarization, $\mathbf{E}_{d}$, is determined by the Poisson equation, $\nabla \cdot\left(\mathbf{E}_{d}+4 \pi \mathbf{P}\right)=0$, which yields $\mathbf{E}_{d}=-4 \pi \mathbf{P} \equiv-4 \pi \mathbf{d}|\psi(\mathbf{r})|^{2}$ (this solution for $\mathbf{E}_{d}$ is definitely a unique one for the spherically symmetric configurations considered below). Finally, the extra term in the GPE induced by the interaction of the local dipole with the collective field, $\mathbf{E}_{d}$, created by all other dipoles is

$$
-\left(\mathbf{d} \cdot \mathbf{E}_{d}\right) \psi \equiv 4 \pi d^{2}|\psi|^{2} \psi
$$

Obviously, this term, if added to Eq. (4), may be absorbed into a redefinition of the effective scattering length, accounting for the repulsion between the particles. In the underlying physical units, this amounts to

$$
a_{s} \rightarrow\left(a_{s}\right)_{\mathrm{eff}} \equiv a_{s}+m d^{2} / \hbar^{2},
$$

where $m$ is the mass of the dipolar molecule. In particular, for the typical value of $a_{s} \sim 10 \mathrm{~nm}$ and the above-mentioned mass of the particle, $\sim 10$ proton masses, Eq. (8) demonstrates that the additional term is essential for the dipole moments $d \gtrsim 1$ Debye (for instance, for water molecules).

Unlike the quantization, the inclusion of the nonlinearity does not break the underlying scaling invariance of the Hamiltonian, cf. Eq. (1). Indeed, under the combined transformation,

$$
\mathbf{r} \rightarrow \alpha \mathbf{r}, \quad \psi \rightarrow \alpha^{-1} \psi, \quad \Omega \rightarrow \alpha^{-2} \Omega .
$$

the total energy of the condensate described by GPE (4),

$$
E_{D}=\frac{1}{2} \int\left[|\nabla \psi|^{2}-\left(U_{0} r^{-2}-\Omega^{2} r^{2}\right)|\psi|^{2}+\left|\psi^{4}\right|\right] d \mathbf{r},
$$

features scaling $E \rightarrow E / \alpha^{4-D}$, where $D$ is the dimension (three or two).

It is relevant to mention that a quantum anomaly was very recently predicted in a model described by the GPE in the 2D space for a harmonically trapped gas of bosons interacting through the two-dimensional repulsive $\delta$-functional potential [16]. The anomaly breaks the specific scaling invariance of this gas, which holds in the mean-field approximation [17].

The rest of the article is organized as follows. In Sec. II, we briefly recapitulate the description of the $3 \mathrm{D}$ and $2 \mathrm{D}$ collapses in the framework of quantum mechanics, extending it through the inclusion of self-similar nonstationary solutions, in addition to the known stationary ones. Section III reports the basic results obtained in the 3D case, which demonstrate the creation of the previously missing GS by the self-repulsive cubic nonlinearity at $U_{0}>1 / 4$, as well as making the GS normalizable at $U_{0}<1 / 4$. It is demonstrated too that, in the latter case, the inclusion of the harmonic trapping potential gives rise to a tristability of bound states. The results obtained in the 2D model with the quintic repulsive term are reported in Sec. IV. In that case, the GS also replaces the quantum-collapse regime for $U_{0}>0$. In the case of $0<-U_{0}<1 / 4$ (i.e., in the case of the weakly repulsive potential), the quintic nonlinearity also gives rise to the GS with a normalizable wave function. In the latter case, three confined modes are found in the presence of the harmonic trap, like in the $3 \mathrm{D}$ setting, but the middle mode is unstable, spontaneously transforming itself into a breather. The article is concluded by Sec. V.

\section{THE COLLAPSE IN QUANTUM MECHANICS}

In the framework of Eq. (2), 3D and 2D stationary states, with angular quantum numbers $l$ and $m$, are looked for as

$$
\begin{gathered}
\psi_{3 \mathrm{D}}=\exp (-i \mu t) Y_{l m}(\theta, \varphi) \phi(r), \\
\psi_{2 \mathrm{D}}=\exp (-i \mu t+i l \varphi) \phi(r),
\end{gathered}
$$


where $\theta$ and $\varphi$ are the angular coordinates and $Y_{l m}(\theta, \varphi)$ is the spherical harmonic. Then, an exact $3 \mathrm{D}$ wave function in the form of Eq. (11) can be readily found, as a solution to Eq. (2), for $U_{l} \equiv U_{0}-l(l+1)<1 / 4$, cf. Ref. [4]:

$$
\begin{gathered}
\phi(r)=\phi_{0} r^{-\sigma_{ \pm}} \exp \left(-\Omega r^{2} / 2\right), \\
\mu=\Omega\left(\frac{3}{2}-\sigma_{ \pm}\right), \quad \sigma_{ \pm} \equiv \frac{1}{2} \pm \sqrt{\frac{1}{4}-U_{l},}
\end{gathered}
$$

the GS corresponding to the smaller value of $\mu$, i.e., $\sigma_{+}$. This solution is also relevant for $U_{l}<0$ (the repulsive potential), with the respective norm,

$$
N_{3 \mathrm{D}}=4 \pi \int_{0}^{\infty} \phi^{2}(r) r^{2} d r
$$

converging (for $\sigma_{+}$) if $U_{l}>-3 / 4$.

In two dimensions, the GS solution to Eq. (2) exist only for $U_{l} \equiv U_{0}-l^{2}<0$, in the exact form given by Eqs. (12) and (13), but with

$$
\mu=\Omega\left(1-\sigma_{ \pm}\right), \quad \sigma_{ \pm}= \pm \sqrt{-U_{l}},
$$

cf. Eq. (14). Like in the 3D case, the GS corresponds to $\sigma_{+}$, the $2 \mathrm{D}$ norm of this solution,

$$
N_{2 \mathrm{D}}=2 \pi \int_{0}^{\infty} \phi^{2}(r) r d r
$$

converging if $U_{l}>-1$.

Past the critical point, i.e., for $U_{l}>1 / 4$ in $3 \mathrm{D}$, and for any $U_{l}>0$ in $2 \mathrm{D}$, the asymptotic form of solutions for $\phi(r)$ at $r \rightarrow 0$ is [2]

$\phi(r) \approx \phi_{0}\left\{\begin{array}{l}r^{-1 / 2} \cos \left[\sqrt{U_{l}-1 / 4} \ln \left(r / r_{0}\right)\right], \quad D=3, \\ \cos \left[\sqrt{U_{l}} \ln \left(r / r_{0}\right)\right], \quad D=2,\end{array}\right.$

with arbitrary constants $\phi_{0}$ and $r_{0}$ (these stationary solutions are exact but unnormalizable ones for $\mu=\Omega=0$ ). The infinite number of radial nodes (zeros) in solutions (18) implies the nonexistence of the GS in these cases and represents the phenomenon of the quantum anomaly. Accordingly, the nonstationary wave function obeying Eq. (2) is expected to collapse, eventually. The latter conjecture was confirmed by direct simulations. As an example, in Fig. 1 we display results

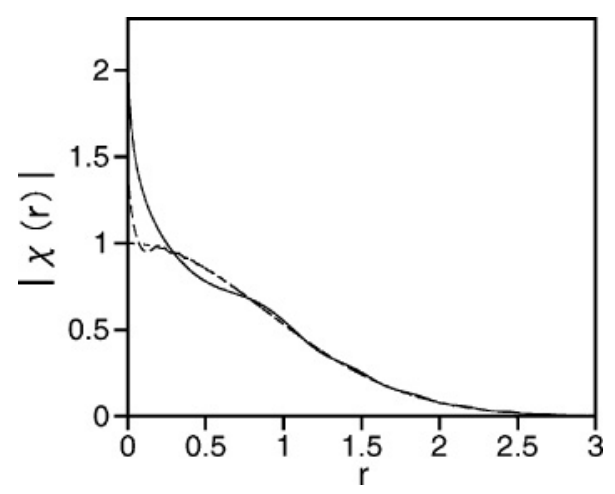

FIG. 1. Three radial profiles of $|\chi(r, t)|=|\psi(r)| r^{1 / 2}$ at $t=0$, 0.005 , and 0.1 (dotted, dashed, and solid curves), as produced by the numerical solution of the radial version of Eq. (2) in 3D with $U_{0}=0.27$ and $\Omega^{2}=0.1$. Note that this value of $U_{0}$ slightly exceeds the critical one, $\left(U_{0}\right)_{\mathrm{cr}}^{(3 \mathrm{D})}=1 / 4$. of the simulations of the 3D spherically symmetric solution, with $l=m=0$. The simulations were performed by means of the split-step Fourier method using $2^{16}$ modes. As the initial condition, we took $\psi(r)=r^{-1 / 2} \exp \left(-\Omega r^{2} / 2\right)$, which is the exact stationary wave function for $U_{0}=1 / 4$, i.e., the one at the critical point, see Eqs. (13) and (14). The snap-shot profiles of $r^{1 / 2}|\psi(r)|$ shown in Fig. 1 testify to the rapid growth of the amplitude of the solution at $r=0$, which is a signature of the development of the collapse. The growth eventually ceases in the simulations, due to the finite mesh size of the numerical scheme.

A more general version of asymptotic solution (18) can be obtained by means of substitution

$$
\psi(r, t)=r^{-1 / 2} Y_{l m}(\theta, \varphi) \chi(r, t)
$$

which makes Eq. (2) for the 3D time-dependent wave function equivalent to its $2 \mathrm{D}$ counterpart:

$i \chi_{t}=-(1 / 2)\left[\partial_{r}^{2}+r^{-1} \partial_{r}+\left(U_{l}-1 / 4\right) r^{-2}-\Omega^{2} r^{2}\right] \chi$

Then, omitting the term $\sim \Omega^{2}$, time-dependent solutions to Eq. (20) may be looked for in a self-similar form, $\chi(r, t)=$ $\chi(\Lambda)$, where

$$
\Lambda \equiv \ln \left(\left(\rho_{0}^{2} / r^{2}\right)\left(t / \tau_{0}\right)\right)
$$

with arbitrary radial and temporal scales, $\rho_{0}$ and $\tau_{0}$. This substitution transforms Eq. (20) with $\Omega=0$ into an ordinary differential equation,

$$
i e^{-\Lambda} \frac{d \chi}{d \Lambda}=-\frac{\tau_{0}}{2 \rho_{0}^{2}}\left[4 \frac{d^{2} \chi}{d \Lambda^{2}}+\left(U_{l}-\frac{1}{4}\right) \chi\right] .
$$

Explicit solutions to Eq. (22) can be found in an asymptotic form, corresponding to $\Lambda \rightarrow \infty$ (i.e., $r \rightarrow 0$ and/or $t \rightarrow \infty$ ), which is a spatiotemporal generalization of the stationary solution (18):

$$
\begin{aligned}
\chi= & \phi_{0} \cos \left((1 / 2) \sqrt{U_{l}-1 / 4} \Lambda\right) \\
& +i \phi_{0} \frac{\sqrt{U_{l}-1 / 4}}{4 U_{l}+3} \frac{r^{2}}{t}\left[\sin \left((1 / 2) \sqrt{U_{l}-1 / 4} \Lambda\right)\right. \\
& \left.+\sqrt{U_{l}-1 / 4} \cos \left((1 / 2) \sqrt{U_{l}-1 / 4} \Lambda\right)\right],
\end{aligned}
$$

the second term being a small correction to the first one. Through substitution (19), solution (23) pertains to the 3D case, and, on the other hand, expression (23) with $\sqrt{U_{l}-1 / 4}$ replaced by $\sqrt{U_{l}}$ directly applies to the $2 \mathrm{D}$ situation.

With regard to Eq. (21), the meaning of solution (23) in both the 3D and 2D cases may be understood by noting that the number of nodes of the wave function [i.e., the number of the corresponding zeros of $\left.\cos \left((1 / 2) \sqrt{U_{l}-1 / 4} \Lambda\right)\right]$ which pass a point with fixed $r$ grows with time as $n \approx$ $\left[\sqrt{U_{l}-1 / 4} /(2 \pi)\right]\left[\ln \left(r_{0}^{2} / r^{2}\right)+\ln \left(t / t_{0}\right)\right]$. This actually implies the development of the quantum-anomaly structure in time, which expands outwards. 


\section{THE 3D GROUND STATE CREATED BY THE CUBIC SELF-REPULSIVE NONLINEARITY}

\section{A. The ground state in the absence of the external trap}

Spherically symmetric stationary solutions to the GPE in the form of Eq. (4) are looked for as

$$
\psi(r, t)=e^{-i \mu t} r^{-1} \chi_{3 \mathrm{D}}(r),
$$

with real function $\chi$ obeying equation

$$
\mu \chi_{3 \mathrm{D}}=-\frac{1}{2}\left[\chi_{3 \mathrm{D}}^{\prime \prime}+\left(U_{0} r^{-2}-\Omega^{2} r^{2}\right) \chi_{3 \mathrm{D}}\right]+r^{-2} \chi_{3 \mathrm{D}}^{3} .
$$

The expansion of solutions to Eq. (25) at $r \rightarrow 0$ is

$$
\chi_{3 \mathrm{D}}(r)=\sqrt{U_{0} / 2}+\chi_{1} r^{s / 2}, \quad s=1+\sqrt{1+8 U_{0}},
$$

where $\chi_{1}$ is a free constant. For any $U_{0}>0$, the cubic nonlinearity supports finite-norm states without the help of the external trapping potential $(\Omega=0)$. Indeed, in this case the asymptotic form of the solution with $\mu<0$ at $r \rightarrow \infty$ is $\chi_{3 \mathrm{D}}=\chi_{0} \exp (-\sqrt{-2 \mu} r)$, with some constant $\chi_{0}$. Combining it with the asymptotic form (26) valid at $r \rightarrow 0$, one may use, as the simplest analytical approximation, the following interpolation for the 3D modes which represent the GS with given norm $N$ :

$$
\psi_{3 \mathrm{D}}^{(\Omega=0)}=\sqrt{\frac{U_{0}}{2}} e^{-i \mu t} r^{-1} e^{-\sqrt{-2 \mu} r}, \quad \mu=-\frac{1}{2}\left(\frac{\pi U_{0}}{N_{3 \mathrm{D}}}\right)^{2} .
$$

The small term $\chi_{1} r^{s / 2}$ from Eq. (26) is not included into this approximation, and the norm was calculated by the substitution of analytical expression (27) into integral (15). Actually, in the limit of $\mu \rightarrow-0$, Eq. (27) gives an asymptotically exact solution (rather than being simply an interpolation), and its limit form corresponding to $\mu=0$ and $N=\infty$ is an exact (although unnormalizable) solution.

Numerical solutions of Eq. (25) were found by means of the shooting method. A typical example of the GS solution, along with the respective approximation (27), is displayed in Fig. 2(a) for $U_{0}=0.8$, which is essentially larger than the critical attraction strength, $\left(U_{0}\right)_{\mathrm{cr}}^{(3 \mathrm{D})}=1 / 4$, beyond which the
GS does not exist in the framework of the linear Schrödinger equation (2). Further, Figs. 2(b) and 2(c) represent the entire family of the solutions for two values, $U_{0}=0.8$ and 0.1 , which are, respectively, larger and smaller than $1 / 4$. The conclusion is that, in the nonlinear model, the GS exists for all values of $U_{0}$ and $N$. Thus, the self-repulsive cubic term completely suppresses the quantum collapse in the 3D space and offers an alternative solution to the quantum-anomaly problem [1], by creating the GS where it does not exist in the linear Schrödinger equation.

The analytical approximation (27) suggests an estimate for the radial size of the GS created by the repulsive nonlinearity:

$$
R_{\mathrm{GS}}^{(3 \mathrm{D})} \equiv \frac{4 \pi}{N_{3 \mathrm{D}}} \int_{0}^{\infty}\left|\psi_{3 \mathrm{D}}^{(\Omega=0)}(r)\right|^{2} r^{3} d r=\frac{N_{3 \mathrm{D}}}{2 \pi U_{0}} .
$$

It is relevant to rewrite this estimate in terms of the physical units, as per Eqs. (5), (6), and (8):

$$
\left[R_{\mathrm{GS}}^{(3 \mathrm{D})}\right]_{\mathrm{ph}} \equiv r_{0} R_{\mathrm{GS}}=\frac{2\left(\hbar^{2} a_{s}+m d^{2}\right) N_{\mathrm{ph}}}{m\left(U_{0}\right)_{\mathrm{ph}}},
$$

which gives the radius of the GS in terms of the physical parameters of the model. Note that arbitrary spatial scale $r_{0}$, which was used in rescalings (5) and (6), does not appear in Eq. (6) (actually, it cancels out). It is natural that $R_{\mathrm{GS}}$ shrinks to zero at $N \rightarrow 0$, which implies the transition to the collapse in the framework of the linear Schrödinger equation. It is worthy to note, too, that, if the term induced by the dipole-dipole interactions dominates over the one induced by the contact interactions ( $m d^{2} \gtrsim \hbar^{2} a_{s}$ ), expression (29) strongly simplifies, taking into account Eq. (3): $\left[R_{\mathrm{GS}}^{(3 \mathrm{D})}\right]_{\mathrm{ph}}=(d /|Q|) N_{\mathrm{ph}}$. For $Q$ taken as the elementary charge, the dipole momentum $d \sim 1$ Debye, and the number of atoms $\sim 10^{5}$, the latter estimates predicts a relatively dense ground state with the radius $\sim 2 \mu \mathrm{m}$.

The existence of the GS with the finite norm at $U_{0}<$ $1 / 4$ in the nonlinear model with $\Omega=0$ is worthy noting, too, as the corresponding stationary solutions to the linear (a)

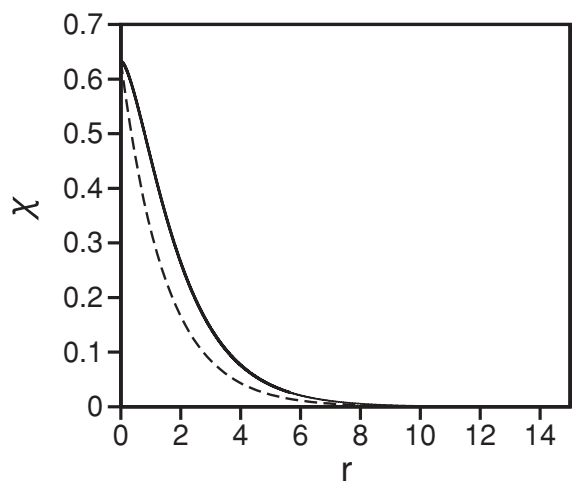

(b)

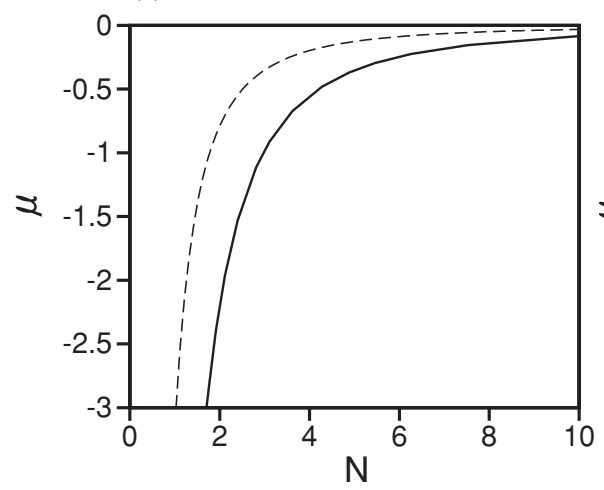

(c)

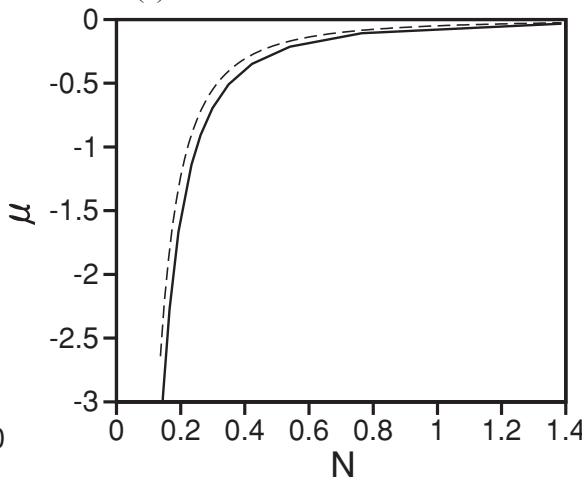

FIG. 2. (a) A typical example of the ground state, shown in terms of $\chi(r) \equiv r|\psi(r)|$, in the 3D nonlinear model without the external trap $(\Omega=0)$, for $U_{0}=0.8$ and $\mu=-0.225$. Panels (b) and (c) display curves $\mu(N)$ for the ground-state families with $U_{0}=0.8$ and 0.1 , respectively. In all the panels, the solid and dashed curves depict the numerical results and analytical approximation (27). In particular, the latter predicts $N(\mu=-0.225)=5.30$ for $U_{0}=0.8$ [the case shown in (a)], while the numerically found counterpart of this value is $N_{\text {num }}(\mu=-0.225)=6.26$. The convergence of the numerical and analytical curves for $N(\mu)$ at $\mu \rightarrow-\infty$ corresponds to the fact that Eq. (27) gives an asymptotically exact solution in this limit. 
equation, see Eqs. (11) and (13), are unnormalizable for $\Omega=0$. Last, simulations of the radial version of Eq. (4) with arbitrary random perturbations added to the stationary solutions (not shown here) demonstrate that the entire GS family is stable. Those simulations did not test the stability of the GS against tesseral (spherical-symmetry-breaking) perturbations, but the repulsive character of both the contact and dipole-dipole interactions (in the mean-field approximation) makes the presence of a symmetry-breaking instability implausible.

It is relevant to mention that, as follows from Eq. (26), the (scaled) energy of the GS, calculated, as per definition (10), in a regularized form, i.e., for $r \geqslant \varrho \rightarrow 0$, contains a diverging term,

$$
\tilde{E}_{3 \mathrm{D}}=\pi U_{0}\left(2-U_{0}\right)(2 \varrho)^{-1},
$$

which may be removed by means of the renormalization, cf. Refs. [1,7]. The vanishing of $\tilde{E}_{3 \mathrm{D}}$ at $U_{0}=2$ seems to be a formal peculiarity rather than a physical feature of the model.

\section{B. Effects of the harmonic trap: the tristability}

The addition of the harmonic trap deforms the GS family reported above for $\Omega=0$. As shown in Fig. 3, at $U_{0}<1 / 4$ the nonlinear model with $\Omega>0$ supports two additional families of 3D confined modes, thus featuring a tristability. The lowest curve in Fig. 3(a) represents the deformed GS branch produced by the numerical solution of Eq. (25), while two upper branches represent a nonlinear deformation of the exact solutions generated by linear equation (2) in the form of Eqs. (11), (13), and (14) (note that in the linear limit, i.e., at $N \rightarrow 0$, the GS created by the nonlinearity disappears by falling to $\mu \rightarrow-\infty)$. The upper branches merge and disappear at $U_{0} \rightarrow 1 / 4$. The stability of all the three families was verified by direct simulations of the radial version of Eq. (4) (not shown here). In the limit of large $N$, all the three branches in Fig. 3(a) asymptotically approach an expression predicted by the Thomas-Fermi approximation,

$$
\left(N_{\mathrm{TF}}\right)_{3 \mathrm{D}} \approx\left(16 \sqrt{2} \pi / 15 \Omega^{3}\right) \mu^{5 / 2} .
$$

(a)
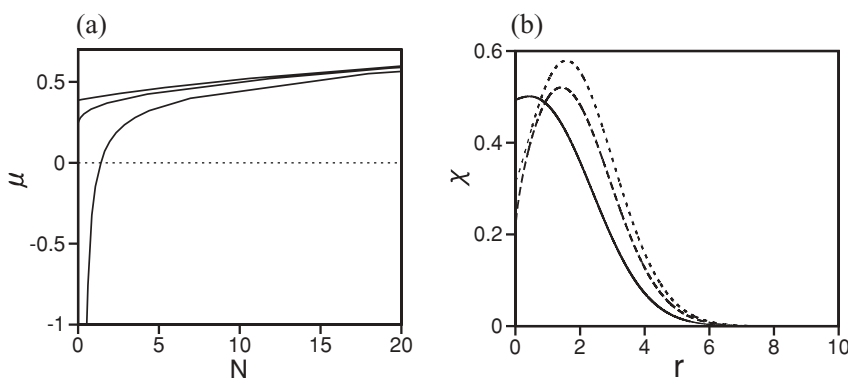

FIG. 3. (a) Curves $\mu(N)$ for three families of the 3D confined modes at $\Omega^{2}=0.1$ and $U_{0}=0.2$. (b) Profiles of typical modes belonging to the upper, middle, and lower branches in (a) are shown by the solid, dashed, and dotted curves, respectively. In panel (b), $\chi \equiv r|\psi(r)|$ for the solution belonging to the lower branch in (a), while $\chi \equiv r^{\sigma_{ \pm}}|\psi(r)|$ for the two upper ones, with the same $\sigma_{ \pm}$as in Eq. (14). The profiles are shown in (b) for $N=10.2$, the respective values of $\mu$ being $0.517,0.503$, and 0.460 .

\section{2D GROUND STATES CREATED BY THE QUINTIC SELF-REPULSIVE NONLINEARITY}

\section{A. The ground state in the absence of the external trap}

As said above, the GPE in the form of Eq. (4) may be relevant in the $2 \mathrm{D}$ case too. However, the $2 \mathrm{D}$ norm of the solution with asymptotic form $\sim r^{-1}$ at $r \rightarrow 0$, which follows from this equation [see Eq. (27)], diverges. In other words, the cubic self-repulsion is not strong enough to prevent the collapse in the 2D setting. On the other hand, the GPE may also include the quintic repulsive term accounting for threebody collisions, provided that the collisions do not give rise to conspicuous losses [18]. The latter assumption may not be quite realistic [19], but, nevertheless, the consideration of the 2D model with the quintic term makes sense, as it is interesting to compare the results with those reported above for the 3D setting.

The 2D axisymmetric GPE with the dominating quintic term is

$$
i \psi_{t}=-(1 / 2)\left(\psi_{r r}+r^{-1} \psi_{r}+U_{l} r^{-2}-\Omega^{2} r^{2}\right) \psi+|\psi|^{4} \psi,
$$

[recall $U_{l} \equiv U_{0}-l^{2}$, if vorticity $l$ is present, see Eq. (12); unlike the $3 \mathrm{D}$ case, the vorticity may be kept in the $2 \mathrm{D}$ analysis based on radial equations]. In Eq. (32), we neglect inessential cubic terms, including the one which may be generated by the dipole-dipole interactions, as in Eq. (8). If the cubic terms are kept, they do not significantly affect the results presented below [in particular, they do not alter the first term in expansion (36) at $r \rightarrow 0]$.

The total energy of the BEC described by $2 \mathrm{D}$ equation (32) is

$E_{2 \mathrm{D}}=\frac{1}{2} \int_{0}^{\infty}\left[|\nabla \psi|^{2}-\left(U_{0} r^{-2}-\Omega^{2} r^{2}\right)|\psi|^{2}+\frac{2}{3}\left|\psi^{6}\right|\right] d \mathbf{r}$,

cf. Eq. (10). This energy features the invariance with respect to the scaling transformation, $E_{2 \mathrm{D}} \rightarrow E_{2 \mathrm{D}} / \alpha$, with the difference from the $3 \mathrm{D}$ case in that the wave function is transformed as per $\psi \rightarrow \alpha^{-1 / 2} \psi$, cf. Eq. (9).

Stationary solutions to Eq. (32) are looked for as

$$
\psi(r, t)=e^{-i \mu t} r^{-1 / 2} \chi_{2 \mathrm{D}}(r),
$$

cf. Eq. (24), which yields an equation for $\chi(r)$ :

$$
\mu \chi_{2 \mathrm{D}}=-\frac{1}{2}\left[\chi_{2 \mathrm{D}}^{\prime \prime}+\left(U_{l}+\frac{1}{4}\right) r^{-2} \chi\right]+r^{-2} \chi^{5},
$$

where we set $\Omega=0$, cf. Eq. (25) in 3D. The expansion of the solution to Eq. (35) at $r \rightarrow 0$ is

$$
\chi=\left[\frac{1}{2}\left(U_{l}+\frac{1}{4}\right)\right]^{1 / 4}+\chi_{1} r^{s},
$$

where $s=(1 / 2)\left(1+\sqrt{5+16 U_{l}}\right)$, and $\chi_{1}$ is an arbitrary constant, cf. Eq. (26) in the $3 \mathrm{D}$ case. The solution with a finite norm exists at $U_{l}>-1 / 4$, representing, at $U_{l}>0$, the suppression of the collapse and creation of the GS by the quintic nonlinearity.

As concerns the vorticity, in the usual situation the amplitude of the corresponding mode must vanish at point $r=0$, where the phase cannot be defined. However, in the present case the solution features a different solution to the 
phase-singularity problem: instead of vanishing, the amplitude diverges at $r \rightarrow 0-$ as $|\psi(r)| \approx\left[(1 / 2)\left(U_{l}+1 / 4\right)\right]^{1 / 4} r^{-1 / 2}$, according to Eqs. (34) and (36). This possibility is akin to the fact that the Bessel equation for amplitudes of vortex modes with topological charge $l$ gives rise to two solutions, $J_{ \pm|l|}($ const $\times r)$, the one with index $-|l|$ being usually omitted as a singular one. However, in the present case the singular solution is acceptable, as it provides for the convergence of $2 \mathrm{D}$ norm (17).

The energy of the GS, if calculated at $r \geqslant \varrho \rightarrow 0$, contains a divergent term, $\tilde{E}_{2 \mathrm{D}}=\pi \sqrt{U_{l}+1 / 4}\left(1-2 U_{l}\right)(3 \sqrt{2} \varrho)^{-1}$, cf. the similar term (30) in the 3D setting. This term, which may be removed by the renormalization procedure, and its vanishing at $U_{l}=1 / 2$, do not seem to be physically significant features of the model.

It is also relevant to mention that the insertion of the current-carrying filament into the pancake-shaped condensate, or passing an electron beam through it, may be a source of loss. However, the above results demonstrate that the probability of collisions of atoms with the filament of radius $\varrho$ scales as $\varrho$, hence it may be made small enough. In particular, for the pancake of thickness $\sim 3 \mu \mathrm{m}$, a simple estimate demonstrates that the radius of the nondiffracting electron beam can be made as small as $1 \mathrm{~nm}$.

Combining the 2D asymptotic form (36), valid at $r \rightarrow 0$, and its counterpart at $r \rightarrow \infty, \chi_{2 \mathrm{D}} \approx \chi_{0} \exp (-\sqrt{-2 \mu} r)$, and making use of definition (17) for the $2 \mathrm{D}$ norm, we obtain an analytical interpolation formula for the GS family, in the absence of the external trap $(\Omega=0)$ :

$$
\begin{gathered}
\psi_{2 \mathrm{D}}^{(\Omega=0)}=\left[\frac{1}{2}\left(U_{l}+\frac{1}{4}\right)\right]^{1 / 4} e^{-i \mu t} r^{-1 / 2} e^{-\sqrt{-2 \mu} r}, \\
\mu=-\left(U_{l}+\frac{1}{4}\right)\left(\frac{\pi}{2 N_{2 \mathrm{D}}}\right)^{2},
\end{gathered}
$$

cf. approximation (27) in the 3D case. Similar to the situation in the 3D case, Eq. (37) gives an asymptotically exact solution (rather than a mere interpolation) for $\mu \rightarrow-0$ and an exact solution with the infinite norm at $\mu=0$. The approximation (37) makes it possible to define the radial size of the twodimensional GS created by the quintic nonlinearity, cf. Eq. (28) in the $3 \mathrm{D}$ case:

$$
R_{\mathrm{GS}}^{(2 \mathrm{D})} \equiv \frac{2 \pi}{N_{2 \mathrm{D}}} \int_{0}^{\infty}\left|\psi_{2 \mathrm{D}}^{(\Omega=0)}(r)\right|^{2} r^{2} d r=\frac{N_{2 \mathrm{D}}}{\pi \sqrt{2\left(U_{0}+1 / 4\right)}} .
$$

Note that the quintic term supports the GS in $2 \mathrm{D}$ even at $0<-U_{l}<1 / 4$, when the central potential is repulsive. The correctness of this counterintuitive conclusion is corroborated by the above-mentioned fact that the analytical approximation (37) gives the asymptotically exact solution for $\mu \rightarrow 0$, including the case of $0<-U_{l}<1 / 4$.

An example of the stable GS, and curves $\mu(N)$ for the GS families in 2D, are displayed, along with the analytical approximation (37), in Fig. 4. The $\mu(N)$ curves are shown for both signs of the central potential (and $l=0$ ), $U_{0}=-0.18$ and $U_{0}=0.05$. Simulations of perturbed solutions within the framework of the radial version of Eq. (32) confirm the stability of the GS families (not shown here). Although
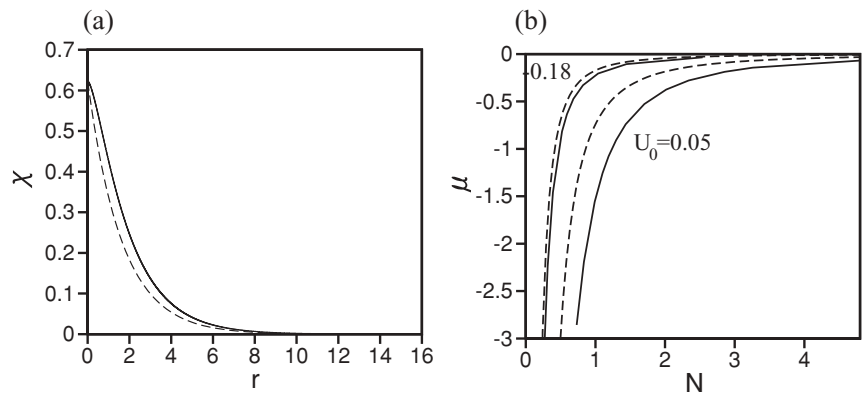

FIG. 4. (a) The radial profile of the ground state in the $2 \mathrm{D}$ model with the quintic nonlinearity and $\Omega=0$ for $U_{0}=0.05$ and $\mu=-0.1867$. (b) Curves $\mu(N)$ for the GS families with $U_{0}=-0.18$ and $U_{0}=0.05$. In both panels, the numerical results and the respective analytical approximation (37) are depicted by the continuous and dashed curves. The convergence of the numerical and analytical curves for $N(\mu)$ at $\mu \rightarrow-0$ corresponds to the fact that Eq. (37) gives an asymptotically exact solution in this limit.

these simulations do not include azimuthal perturbations, the repulsive sign of the nonlinearity makes it evident that these perturbations will not give rise to an instability [20].

\section{B. Effects of the harmonic trap}

As shown in Fig. 5(a), in the presence of the external trap $(\Omega>0)$, three different stationary solutions can be found at $-1 / 4<U_{l}<0$, when the corresponding 2D linear Schrödinger equation gives rise to two exact solutions, in the form given by Eqs. (12), (13), and (16). As well as in the similar situation for the 3D case, which is displayed in Fig. 3(a), two upper branches in Fig. 5 represent nonlinear deformations of the exact linear solutions, with values of $\mu$ approaching those given by Eq. (16) in the limit of $N \rightarrow 0$. The lowest branch represents the GS corresponding to approximation (37), which is additionally deformed by the trapping potential. In the limit of $\mu \rightarrow \infty$, both the bottom and top branches in Fig. 5(a) asymptotically approach the Thomas-Fermi limit, $\left(N_{\mathrm{TF}}\right)_{2 \mathrm{D}} \approx\left(4 \pi / 3 \Omega^{2}\right) \mu^{3 / 2}$, cf. Eq. (31).

In spite of the overall similarity to the $3 \mathrm{D}$ case, it is worthy to note that the shape of the middle branch in Fig. 5(a) differs drastically from its counterpart in the 3D case,
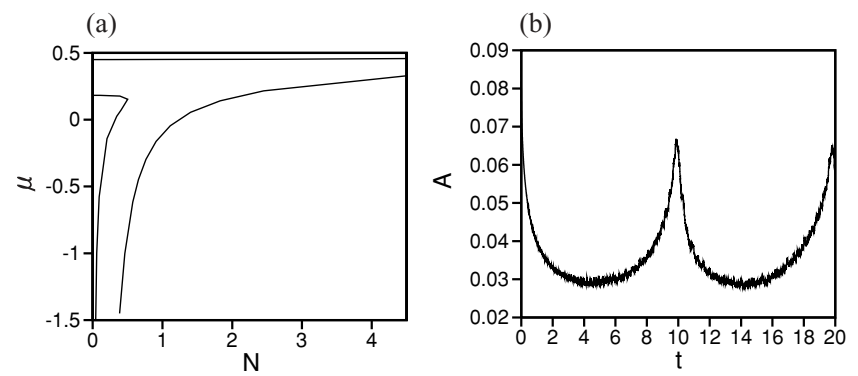

FIG. 5. (a) Curves $\mu(N)$ for three stationary modes found in the $2 \mathrm{D}$ model with the quintic nonlinearity for $U_{0}=-0.18$ and $\Omega^{2}=0.10$, cf. the similar picture for the 3D cubic model displayed in Fig. 3. (b) An example of the breather generated by the solution with $N=0.3453$ belonging to the unstable middle branch from panel (b). The plot shows $A \equiv|\chi(r=0, t)|^{2}$ as a function of time, with $\chi$ defined as $\chi_{2 \mathrm{D}}$ in Eq. (34). 
cf. 3(a). Moreover, direct simulations of Eq. (32) demonstrate that while the solutions corresponding to the top and bottom branches in Fig. 5(a) are stable (not shown here in detail), the middle-branch solutions are not. In the simulations, they are spontaneously transformed into robust breathers featuring long-period oscillations; see an example in Fig. 5(b).

\section{CONCLUSION}

We have demonstrated that the mean-field repulsive nonlinearity suppresses the $3 \mathrm{D}$ quantum collapse induced by the central attractive potential, $-\left(U_{0} / 2\right) r^{-2}$, which can be realized in the ultracold gas of dipolar molecules attracted by the central charge. The dipole-dipole interactions were also taken into account, resulting (in the framework of the mean-field approximation) in a redefinition of the scattering length which accounts for the contact repulsion. The nonlinearity creates the GS (ground state) in place of the collapse regime. For $U_{0}<1 / 4$, when the respective $3 \mathrm{D}$ Schrödinger equation does not lead to the collapse, the inclusion of the harmonic trap gives rise to the tristability. The cubic repulsion is not strong enough to prevent the $2 \mathrm{D}$ collapse, but the quintic term is sufficient for this purpose. It also gives rise to the GS which replaces the quantum collapse in the 2D space and to similar modes carrying the angular momentum (which feature the amplitude diverging, rather than vanishing, at $r \rightarrow 0$, while the total norm of the vortical mode converges). A difference from the
3D case is that, in the presence of the harmonic trap, one of the three confined modes supported by the weakly repulsive central potential with $0<-U_{0}<1 / 4$, in the combination withe quintic nonlinearity, is unstable, transforming itself into a breather. A counterintuitive finding is that the 2D self-trapped mode exists even in the case of the weakly repulsive potential, while the harmonic trap is absent.

This work suggests continuations in several directions. If the orientation of the dipoles in the $3 \mathrm{D}$ space is fixed by an external uniform field, the central charge induces an axisymmetric potential, $U=-\left(U_{0} / 2\right) r^{-2} \cos \theta$, and it may be interesting to study the possibility of the replacement of the corresponding anisotropic collapse (cf. Ref. [7]) by a GS. It is relevant to mention that the dipole-dipole interactions between the bosons may give rise to a specific mode of the nonlinear collapse in BEC [21], in the absence of the contact repulsion, which suggests considering an interplay of this nonlinear mode with the linear collapse (although the dynamics of the collapse is not described by the mean-field approximation [15]). Another interesting extension may be the study of higher-order nonlinear states, such as 3D vortical modes.

A challenging problem is to extend the analysis to fermion gases. In that connection, it is relevant to mention that the fall-onto-the-center effect was studied for interacting fermion pairs in the framework of the Bethe-Salpeter equation [22].
[1] K. S. Gupta and S. G. Rajeev, Phys. Rev. D 48, 5940 (1993); H. E. Camblong, L. N. Epele, H. Fanchiotti, and C. A. García Canal, Phys. Rev. Lett. 85, 1590 (2000); Ann. Phys. (NY) 287, 57 (2001).

[2] L. D. Landau and E. M. Lifshitz, Quantum Mechanics: Nonrelativistic Theory (Nauka, Moscow, 1974).

[3] M. Ávila Aoki, C. Cisneros, R. P. Martínez-y-Romero, H. N. Núñez-Yepez, and A. L. Salas-Brito, Phys. Lett. A 373, 418 (2009).

[4] S.-H. Dong and M. Lozada Cassou, Am. J. Appl. Sci. 2, 376 (2005).

[5] S. Schmid, A. Härter, and J. H. Denschlag, Phys. Rev. Lett. 105, 133202 (2010).

[6] C. Desfrançois, H. Abdoul-Carime, N. Khelifa, and J. P. Schermann, Phys. Rev. Lett. 73, 2436 (1994)

[7] H. E. Camblong, L. N. Epele, H. Fanchiotti, and C. A. García Canal, Phys. Rev. Lett. 87, 220402 (2001).

[8] J. Deiglmayr, A. Grochola, M. Repp, K. Mörtlbauer, C. Glück, J. Lange, O. Dulieu, R. Wester, and M. Weidemüller, Phys. Rev. Lett. 101, 133004 (2008).

[9] S. Ospelkaus, K.-K. Ni, G. Quéméner, B. Neyenhuis, D. Wang, M. H. G. de Miranda, J. L. Bohn, J. Ye, and D. S. Jin, Phys. Rev. Lett. 104, 030402 (2010).

[10] I. Bloch, J. Dalibard, and W. Zwerger, Rev. Mod. Phys. 80, 885 (2008).

[11] A. Griesmaier, J. Werner, S. Hensler, J. Stuhler, and T. Pfau, Phys. Rev. Lett. 94, 160401 (2005); T. Lahaye, C. Menotti, L. Santos, M. Lewenstein, and T. Pfau, Rep. Prog. Phys. 72, 126401 (2009).
[12] Q. Beaufils, R. Chicireanu, T. Zanon, B. Laburthe-Tolra, E. Maréchal, L. Vernac, J.-C. Keller, and O. Gorceix, Phys. Rev. A 77, 061601(R) (2008).

[13] F. Lang, K. Winkler, C. Strauss, R. Grimm, and J. Hecker Denschlag, Phys. Rev. Lett. 101, 133005 (2008).

[14] L. Santos, G. V. Shlyapnikov, P. Zoller, and M. Lewenstein, Phys. Rev. Lett. 85, 1791 (2000).

[15] L. Pitaevskii and S. Stringari, Bose-Einstein Condensation (Clarendon Press, Oxford, 2003).

[16] M. Olshanii, H. Perrin, and V. Lorent, Phys. Rev. Lett. 105, 095302 (2010)

[17] L. P. Pitaevskii and A. Rosch, Phys. Rev. A 55, R853 (1997).

[18] F. K. Abdullaev, A. Gammal, L. Tomio, and T. Frederico, Phys. Rev. A 63, 043604 (2001); F. K. Abdullaev and M. Salerno, ibid. 72, 033617 (2005).

[19] E. A. Burt, R. W. Ghrist, C. J. Myatt, M. J. Holland, E. A. Cornell, and C. E. Wieman, Phys. Rev. Lett. 79, 337 (1997); D. M. Stamper-Kurn, M. R. Andrews, A. P. Chikkatur, S. Inouye, H. J. Miesner, J. Stenger, and W. Ketterle, ibid. 80, 2027 (1998); J. L. Roberts, N. R. Claussen, S. L. Cornish, and C. E. Wieman, ibid. 85, 728 (2000).

[20] B. A. Malomed, D. Mihalache, F. Wise, and L. Torner, J. Optics B: Quantum S. O. 7, R53 (2005).

[21] T. Lahaye, J. Metz, B. Fröhlich, T. Koch, M. Meister, A. Griesmaier, T. Pfau, H. Saito, Y. Kawaguchi, and M. Ueda, Phys. Rev. Lett. 101, 080401 (2008).

[22] G. Tiktopoulos, J. Math. Phys. 6, 573 (1965); G. V. Efimov, Few-Body Syst. 41, 157 (2007). 